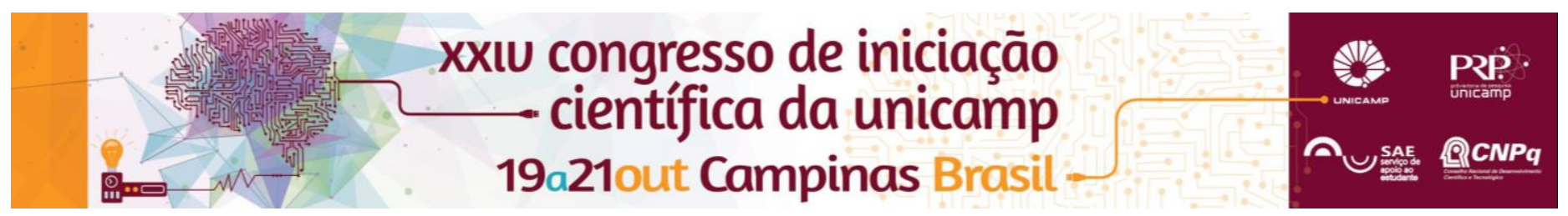

\title{
The Monte Carlo Method and its application to Themodynamics of Ice
}

\section{João Paulo Castro Zerba}

\begin{abstract}
This present project concerns computational simulations utilizing Monte Carlo Method that was used with the onedimensional Ising model of a spin chain, and besides of theoretical study regarding the molecular structure of ice, it was made some development about strategy to implement the calculus of the configurational entropy of hexagonal ice (Ih).
\end{abstract}

Key words: Monte Carlo, Ising, hexagonal ice

\section{Introduction}

The project aims to introduce computer simulation, specifically the Monte Carlo method and the study of the calculation of configurational entropy of I-hexagonal ice.

\section{Results and Discussion}

For a large number of spins on the network even in one dimension, the calculations can be very difficult and long to be estimated, since as each spin site can have two different states, \pm 1 , so there is $2 \mathrm{~N}$ different possible states for the system, motivating computer simulation. With the study of the Monte Carlo method applied to Ising model to calculate the energy and magnetization of a chain of spins, it was understood that, for a onedimensional chain in the absence of external magnetic field, magnetization occurs only at absolute zero.

Using the analytical method developed by Pauling, the value of entropy at absolute zero was obtained, and the study of the implementation of Ih ice was initiated and the topology of the two-dimensional system of ice molecules was determined. But time was insufficient for the implementation and data analysis.

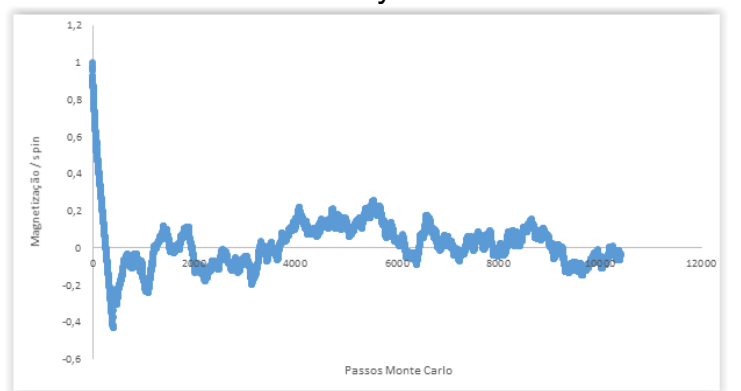

Image 1. Graph magnetization (per spin) by the number of Monte Carlo steps for 1000 spins.

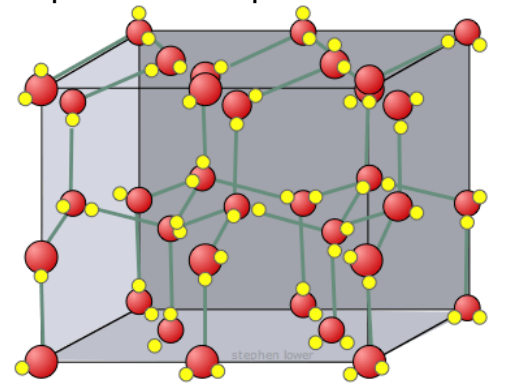

Image 2. Hexagonal ice lattice, bigger balls are oxygen atoms and smaller balls are hydrogen atoms

\section{Conclusions}

The computer simulation is without doubt the most modern and practical way to understand physical problems that in many cases would be complicated to be studied in laboratories. So this branch of physics forward in time in order to make predictions and even confirmations of laws of nature.

The one-dimensional Ising model is a great example of the practical use of the Monte Carlo method, as the analytical results are known and we could compare the results with those predicted by theory.

According to Pauling the calculation of configurational entropy of ice Ih at 0 Kelvin temperature is nonzero. For a two-dimensional system to be simulated we would implement using Reversible Scaling method along with the Monte Carlo method but the period was insufficient for the completion of this final stage.

\section{Acknowledgement}

My thanks to my advisor Dr. Alex Antonelli, to CNPq (Conselho Nacional de Desenvolvimento Científico e Tecnológico) for SAE - UNICAMP (Serviço de Apoio ao Estudante - University of Campinas) and Fapesp (Fundação de Amparo à Pesquisa do Estado de São Paulo).

Reif, F. Berkeley Physics Course; McGraw-Hill: New York, 1967.

Pang, T. An Introduction to Computational Physics, 2nd ed.; Cambridge University Press: Cambridge, 2006.

. Landau, D. P.; Binder, K. A Guide to Monte Carlo Simulations in Statistical Physics; Cambridge University Press: Cambridge, 2000.

Petrenko, V. F.; Whitworth, R. W. Physics of Ice; Oxford University Press: Oxford, 1999.

Koning, M.; Antonelli, A.; Yip, S. Optimized Free-Energy Evaluation Using a Single Reversible-Scaling Simulation. PHYSICAL REVIEW LETTERS 1999, 3973-3977.

Herrero, C. P.; Ramírez, R. Configurational Entropy of Ice from Thermodynamic Integration. Chemical Physics Letters 2013, 568-569, 70-74.

Pauling, L. The Structure and Entropy of Ice and of Other Crystals with Some Randomness of Atomic Arrangement. J. Am. Chem. Soc. Journal of the American Chemical Society 1935, 57 (12), 2680-2684. 